\title{
Interdisciplinary Studies in the Genetic Epidemiology of Cancer
}

National Cancer Institute

\section{Source}

National Cancer Institute. Interdisciplinary Studies in the Genetic Epidemiology of Cancer.

NCl Thesaurus. Code C15941.

Collaborative and interdisciplinary genetic epidemiology investig ations designed to identify and evaluate the interactions of genetic and epidemiologic risk factors leading to cancer susceptibility in individuals, families and populations, and factors influencing the rate of increase with age in cancer susceptibility. Emphasis on multi-site, cooperative research by multi- disciplinary teams of investigators wishing to collaborate within the common theme of the genetic epidemiology of cancer. Particular emphasis is placed on encouraging interdisciplinary, population-based research focusing on the etiologic mechanisms underlying the interaction of genetic and epidemiologic risk factors for cancer susceptibility. The effect on cancer susceptibility of age-related chang es over the lifespan, and the genetic factors leading to older age at onset of cancer are also of interest. 